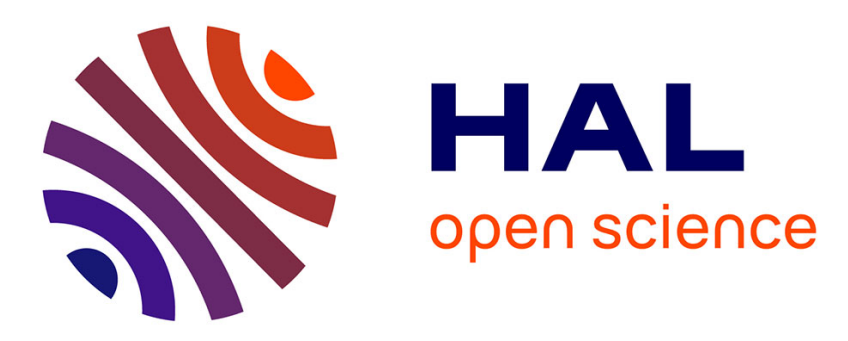

\title{
Ultrasonic and microscopic investigation of blends of polydimethylsiloxane and polyisobutylene at all concentrations
}

\author{
Pierre-Yves Longin, Claude Verdier, Monique Piau
}

\section{To cite this version:}

Pierre-Yves Longin, Claude Verdier, Monique Piau. Ultrasonic and microscopic investigation of blends of polydimethylsiloxane and polyisobutylene at all concentrations. Journal of Rheology, 2000, 44 (5), pp.1189-1203. hal-00322169

\section{HAL Id: hal-00322169 https://hal.science/hal-00322169}

Submitted on 18 Sep 2008

HAL is a multi-disciplinary open access archive for the deposit and dissemination of scientific research documents, whether they are published or not. The documents may come from teaching and research institutions in France or abroad, or from public or private research centers.
L'archive ouverte pluridisciplinaire HAL, est destinée au dépôt et à la diffusion de documents scientifiques de niveau recherche, publiés ou non, émanant des établissements d'enseignement et de recherche français ou étrangers, des laboratoires publics ou privés. 


\title{
Ultrasonic and microscopic investigation of blends of polydimethylsiloxane and polyisobutylene at all concentrations
}

\author{
P.-Y. Longin, C. Verdier, M. Piau \\ Laboratoire de Rhéologie*, \\ BP 53, Domaine Universitaire, 38041 Grenoble, Cedex 09, \\ France
}

Synopsis

PDMS/PIB blends are investigated using ultrasonic techniques ( $\mathrm{MHz}$ range), in the complete concentration range $[0-100 \%]$. The velocities of propagation and attenuations of shear and longitudinal waves are determined experimentally together with simultaneous optical microscopy observations.

From the knowledge of the acoustical and physical properties of the separate polymers, the acoustical longitudinal parameters of the blend can be predicted successfully up to $40 \%$ volume concentration of the dispersed phase, with a model developed by the authors and especially adapted to wave propagation in such viscoelastic emulsions.

For shear waves, two emulsion models (Palierne and Lee-Park) are used for predicting the viscoelastic moduli associated with the acoustical data at ultrasonic frequencies, with a good agreement. The case of composite droplets [40-60\%] has required to consider Friedrich's extension of Palierne's model.

The ultrasonic method appears to be a very interesting tool for predicting phase inversion for polymer blends, as well as the evolution of the microstructure of the blend. Finally, this work provides an extension of the validity of emulsion models in the high frequency range.

Keywords: polymer blend, ultrasound, longitudinal waves, shear waves, optical microscopy, emulsion model

\footnotetext{
* Université Joseph Fourier, Institut National Polytechnique de Grenoble, CNRS (UMR 5520)
} 


\section{INTRODUCTION}

The blending of commercially available polymers is one way to design new polymeric materials with advanced properties. Most of the common polymers are incompatible, so that blending processes give rise to a heterogeneous microstructure characterized at low concentrations by a distribution of inclusions of one polymer (the dispersed phase) embedded in a matrix formed by the other polymer (the continuous phase). At rest or under slow flow, these inclusions are spherical. The processing and ultimate properties of the blends [Utracki (1989)] depend on the size distribution of the inclusions and the interfacial tension between the components. Therefore, it is of great interest to develop methods adapted to the characterization of the blend morphology and to model their rheological behavior.

Characterization of the microstructure is difficult to carry out on line, although it would be important to control the mixing of two polymers during blend processing, in an extruder for instance, through a non-destructive on-line technique. The ultrasonic techniques found multiple uses in different fields of application, in particular to characterize solid and molten polymers [Bridge (1987), Arman (1979), for instance]. Work on the ultrasonic wave propagation in suspensions and emulsions dates 50 years back. In 1948, Urick reported data of the ultrasonic attenuation in aqueous kaolin and sand dispersions. Epstein and Carhart (1953) developed theories for water droplets in air and later Allegra and Hawley (1972) looked at the more general cases of liquid-liquid emulsions and of suspensions. They relate the evolution of the acoustical parameters (velocity of propagation of longitudinal waves, attenuation) to the radius and the volume fraction of the spherical inclusions. These theories are only valid in the long wavelength limit. For wavelengths comparable to the size of the inclusions, resonance effects may appear.

Recently, a technique of investigation of polymer blends has been proposed and tested by Verdier and Piau (1996, 1997). They measured the acoustical parameters of an incompatible blend (with spherical inclusions) in the Megahertz range during extrusion. This was combined with a wave propagation model in viscoelastic emulsions well adapted for such two-phase polymeric materials. 
Investigations undertaken in the last decade to understand the rheological properties of twophase polymer systems mostly use small amplitude oscillatory shear [Scholtz et al. (1989) ; Graebling and Müller (1990); Brahimi et al. (1991); Gramespacher and Meissner (1992); Graebling et al. (1993); Friedrich et al. (1995); Lacroix et al. (1997, 1998); Bousmina (1999)]. This procedure is assumed not to change the microstructure of the blends during the measurements. One characteristic feature of two-phase polymer blends is the appearance of a shoulder in the storage shear modulus $\mathrm{G}^{\prime}$ at low frequencies due to the relaxation of the deformable dispersed particles in the dilute and semi dilute ranges.

Theoretical approaches are proposed in the literature to model the behavior of blends under shear oscillatory flow. The Oldroyd (1953) emulsion model has been refined to account for the viscoelasticity of the components [Graebling and Müller (1990); Palierne (1990)] as well as the radii distribution, with a good agreement with experimental data. It cannot be used for large deformations where the dispersed phase is highly deformed. Lee and Park (1994) have extended the Doi and Ohta theory (1991) for materials with a complex interface to take into account the viscosity mismatch in polymer blends. The Lee and Park is able to describe flows where breakup and coalescence processes take place in a complex rheological manner Recently, Vinckier et al. (1996) used this theory for several blends of nearly inelastic polymers at various concentrations and viscosity ratios.

The aim of this paper is to characterize the morphology of molten polymers blends using complementary ultrasonic techniques and optical microscopy. Longitudinal and shear tests were carried out in PDMS/PIB mixtures at ambient temperature. Blend compositions cover the whole volume concentration range (from 0 to $100 \%$ ). Temperature and frequency were kept constant during the tests. Section II presents the theories used in this work. Section III describes the PDMS/PIB blends as well as the experimental systems used for shear and longitudinal waves. In section IV, the results are described and compared with existing theories. Discussions deal with the applicability of such models and their validity at high frequencies.

\section{THEORY}

\section{A. Longitudinal waves: two phase viscoelastic model}


Theories of longitudinal wave propagation in fluid-based mixtures have been developed by Epstein and Carhart (1953) or Allegra and Hawley (1972) in the case of various emulsions or suspensions of particles in a newtonian fluid. These theories work well at low volume concentrations (up to 10\%), and corrections can be used for higher concentrations [Lloyd and Berry (1967)]. Recently, they were extended to viscoelastic emulsions [see papers by Verdier and Piau (1996, 1997)]. Linear viscoelastic effects are introduced through an integral law of the type:

$$
\Sigma=-p I+\int_{-\infty}^{t}\left\{\tilde{K}\left(t-t^{\prime}\right)-\frac{2}{3} G\left(t-t^{\prime}\right)\right\} \operatorname{Tr} D\left(t^{\prime}\right) \quad I d t^{\prime}+2 \int_{-\infty}^{t} G\left(t-t^{\prime}\right) D\left(t^{\prime}\right) \quad d t^{\prime}
$$

$\Sigma, \mathrm{I}, \mathrm{D}$ are respectively the stress, the identity, and the symmetric part of the velocity gradient tensors. $\tilde{K}(t)$ and $G(t)$ are the compressibility and shear relaxation functions. This model is adequate when considering small deformations, which correspond to the ones encountered in non destructive ultrasonic tests.

From the basic equations, the formula giving the velocity of propagation $v_{L}$ and the attenuation $\alpha_{\mathrm{L}}$ of longitudinal waves takes the form:

$$
\left(\frac{\mathrm{B}}{\mathrm{k}}\right)^{2}=1-\frac{3 \mathrm{i} \varepsilon}{\mathrm{k}^{3} \mathrm{R}^{3}}\left(\mathrm{~A}_{0}+3 \mathrm{~A}_{1}\right)-\frac{27 \varepsilon^{2}}{\mathrm{k}^{6} \mathrm{R}^{6}}\left(\mathrm{~A}_{0} \mathrm{~A}_{1}+2 \mathrm{~A}_{1}^{2}\right)
$$

where $B$ is the complex wave number defined by $B=\left(\omega / v_{L}\right)+i \alpha_{L}$ and $\omega$ is the angular frequency ( $\omega=2 \pi f$, $f$ is the frequency), $\varepsilon$ is the volume fraction of the dispersed phase, $R$ the radius of the inclusions, $\mathrm{k}$ is the complex wave number of the incident wave in the matrix. $\mathrm{A}_{0}$ and $\mathrm{A}_{1}$ are complex coefficients used in the development of the acoustical potential. They are found by solving a system of boundary conditions which arises when a wave impinges onto a spherical obstacle [Verdier and Piau (1997)]. From formula (2), $v_{L}$ and $\alpha_{L}$ can be obtained by taking the real and imaginary parts of $\mathrm{B}$.

This model has the advantage to integrate a complete description of the viscoelastic and thermal wave phenomena involved, as well as the Rayleigh scattering of the incident wave into different directions. Many expressions for the velocity of sound and the attenuation in a two-phase medium may be found in the literature. For particles or droplets suspended in viscous fluids, Urick (1947) gave the simple formula for the velocity:

$$
v_{a}=\left(\kappa_{a} \rho_{a}\right)^{-1 / 2}
$$


where $\rho_{\mathrm{a}}=(1-\varepsilon) \rho_{\mathrm{m}}+\varepsilon \rho_{\mathrm{d}}$ is an average density, $\kappa_{\mathrm{a}}=(1-\varepsilon) \kappa_{\mathrm{m}}+\varepsilon \kappa_{\mathrm{d}}$ is the average compressibility, and lower indexes $\mathrm{m}$ and $\mathrm{d}$ refer respectively to the suspending fluid (matrix) and the dispersed phase. This "average" velocity $v_{a}$ has been used for instance as a reference velocity when studying wave propagation in oil-in-water newtonian emulsions [McClements and Povey (1989)]. Other more complete expressions take into account the viscous dissipation at the interface through the ratio $\left(\delta_{S} / R\right)$, where $\delta_{S}$ is the viscous skin depth [McClements and Povey (1987); Harker and Temple (1988)].

The classical part of the sound absorption in mixtures is an "average" attenuation $\alpha_{a}$ and may be written, when the absorption coefficients of the fluid and the dispersed phases are small [Allegra and Hawley (1972); Kaatze et al. (1996)]:

$$
\alpha_{\mathrm{a}}=\alpha_{\mathrm{m}}+\frac{\varepsilon}{2} \quad\left\{\alpha_{\mathrm{m}}\left(\frac{\rho_{\mathrm{m} \mathrm{v}_{\mathrm{m}}}{ }^{2}}{\rho_{\mathrm{d} \mathrm{d}^{2}}}-3\right)+2 \alpha_{\mathrm{d}} \frac{\rho_{\mathrm{m}} \mathrm{v}_{\mathrm{m}}}{\rho_{\mathrm{d} \mathrm{v}_{\mathrm{d}}}}\right\}
$$

The so-called (damped) thermal waves and viscous waves, generated at the interface give rise to contributions to the total attenuation coefficient that will also alter $\alpha_{a}$. These contributions are known to reach noticeable values when the corresponding thermal and viscous skin depths $\delta_{\mathrm{T}}$ and $\delta_{\mathrm{S}}$ respectively are of the same order as the particle size. At small concentrations (lower than $10 \%$ ), the different contributions can be separated. But in the more general case of viscoelastic systems at moderate concentrations, their combination can become rather intricate and a complete model like the present one is needed.

In this model, the expression for $\delta_{\mathrm{T}}$ is the usual one:

$$
\delta_{\mathrm{T}}=\left(\frac{2 \tau}{\rho \mathrm{C}_{\mathrm{p}} \omega}\right)^{1 / 2}
$$

where $\tau$ is the thermal conductivity, $C_{p}$ is the specific heat at constant pressure.

The expression for $\delta_{\mathrm{S}}$ takes the generalized form :

$$
\delta_{\mathrm{S}}=\frac{\left|\mathrm{G}^{*}\right|}{\omega \mathrm{G}^{\prime \prime}}\left\{\frac{2\left(\mathrm{G}^{\prime}+\mathrm{G}^{*} \mid\right)}{\rho}\right\}^{1 / 2}
$$

which reduces to the usual one $(2 \eta / \rho \omega)^{1 / 2}$ in the case of the newtonian fluid (viscosity $\left.\eta\right)$.

\section{B. Shear waves: shear models for emulsions}

The model used for longitudinal waves was developed assuming that the wavelength is larger than the droplet size (long wave regime). For the blends tested which are in the melt state, shear wave velocities are found to range from 40 to $140 \mathrm{~m} / \mathrm{s}$. Shear wavelengths range between 16 and $60 \mu \mathrm{m}$ and are of the same order as the inclusion radii, which is 
incompatible with the previous model assumptions. Furthermore, no shear wave propagation model is available in the literature for such two-phase viscoelastic fluids. Therefore well-known emulsion models will be considered to test their abilities to predict experimental data in the whole range of concentrations, at frequencies in the $\mathrm{MHz}$ range.

The complete formulae giving the complex modulus $\mathrm{G}_{\mathrm{b}}{ }^{*}(\omega)$ of a blend at low frequencies enclose the interfacial tension $\sigma$ and the radius $R$ through functions of the ratio $\sigma / R$. At high frequencies, the interfacial tension contribution is actually found to be negligible and this ratio disappears so that $\mathrm{R}$ does not show in the expressions of $\mathrm{G}_{\mathrm{b}}{ }^{*}(\omega)$.

\section{Palierne model (1990)}

The model proposed by Palierne (1990) has been shown to be relevant for predicting the rheological behavior of various immiscible blends even at relatively high concentrations of the dispersed phase and at frequencies as high as $10 \mathrm{~Hz}$ [Lacroix et al. (1997)]. It has never been compared with measurements at ultrasonic frequencies. In this limiting case, $\mathrm{G}_{\mathrm{b}}{ }^{*}(\omega)$ may be written as follows :

$$
\mathrm{G}_{\mathrm{b}}{ }^{*}(\omega)=\frac{1+\frac{3}{2} \mathrm{H}^{*}(\omega)}{1-\mathrm{H}^{*}(\omega)} \quad \mathrm{G}_{\mathrm{m}}{ }^{*}(\omega)
$$

where $H^{*}(\omega)$ is defined by:

$$
H^{*}(\omega)=\varepsilon \frac{2 G_{d}^{*}(\omega)-2 G_{m}^{*}(\omega)}{2 G_{d}^{*}(\omega)+3 G_{m}^{*}(\omega)}
$$

$\mathrm{G}_{\mathrm{m}}{ }^{*}(\omega)$ and $\mathrm{G}_{\mathrm{d}}{ }^{*}(\omega)$ are the complex moduli of the matrix and dispersed phase respectively.

\section{Lee-Park model (1994)}

This model was developed by Lee and Park to extend the Doi-Ohta theory, initially derived for a blend of two immiscible newtonian fluids with the same viscosities and densities, to viscoelastic fluids of different viscosities and densities over a large range of frequencies. At high frequencies, the complex modulus $\mathrm{G}_{\mathrm{b}}{ }^{*}(\omega)$ of the blend is expressed by :

$$
\mathrm{G}_{\mathrm{b}}{ }^{*}(\omega)=\left(1+\varepsilon \frac{3 \mathrm{G}_{\mathrm{d}}{ }^{*}(\omega)-3 \mathrm{G}_{\mathrm{m}}{ }^{*}(\omega)}{5 \mathrm{G}_{\mathrm{d}}{ }^{*}(\omega)+5 \mathrm{G}_{\mathrm{m}}{ }^{*}(\omega)}\right) \mathrm{G}_{\mathrm{m}}{ }^{*}(\omega)
$$

The right-hand term in (9) was derived from a mixing rule which accounts for the polymer viscosities mismatch. The behavior of PS/PE and PETG/EVA mixtures was found to be correctly described by this model even at high frequencies $(1-10 \mathrm{~Hz})$ by Lacroix et al. (1997). On the contrary, these authors observed discrepancies between the Lee-Park model 
and their data for blends with components with a low viscosity ratio (PP/EVA with a zero shear-viscosity ratio $\eta_{d} / \eta_{m}$ around 0.1 ).

\section{Friedrich model}

Palierne's model was recently extended to the case of composite droplets by the group of Friedrich $(1995,1999)$. This extension predicts the shear moduli of the blend for low and moderate frequencies. A composite droplet system A-B is made of droplets of polymer B inside another polymer A. The droplets B can also contain smaller droplets of polymer A. Denoting by :

$\phi_{1}$ the volume concentration of small droplets A inside a composite droplet B,

$\phi_{2}$ the volume concentration of composite droplets B inside the matrix A,

$\phi_{3}$ the volume concentration of empty droplets B inside the matrix A,

$\mathrm{GA}_{\mathrm{A}} *(\omega)$ and $\mathrm{GB}^{*}(\omega)$ the complex shear moduli of polymer $\mathrm{A}$ and $\mathrm{B}$,

the resulting modulus of the blend $\mathrm{G}_{\mathrm{b}} *(\omega)$ for high frequencies (limiting form neglecting interfacial tension effects) is:

$$
\mathrm{G}_{\mathrm{b}} *(\omega)=\mathrm{G}_{\mathrm{A}} *(\omega) \frac{1+3\left(\phi_{2} \mathrm{H}_{2}(\omega)+\phi_{3} \mathrm{H}_{3}(\omega)\right)}{1-2\left(\phi_{2} \mathrm{H}_{2}(\omega)+\phi_{3} \mathrm{H}_{3}(\omega)\right)}
$$

where

$$
\mathrm{G}_{\mathrm{C}} *(\omega)=\mathrm{G}_{\mathrm{B}} *(\omega) \frac{1+3 \phi_{1} \mathrm{H}_{1}(\omega)}{1-2 \phi_{1} \mathrm{H}_{1}(\omega)}
$$

$\mathrm{H}_{1}(\omega), \mathrm{H}_{2}(\omega)$ and $\mathrm{H}_{3}(\omega)$ are functions of $\mathrm{G}_{A} *(\omega), \mathrm{GB}^{*}(\omega)$ and $\mathrm{GC}^{*}(\omega)$

\section{EXPERIMENTAL PART}

The aim of this study is to check the accuracy of the method with model emulsions, that means well-defined products and simple interfaces, at all concentrations. Previous studies carried out by the authors [Verdier and Piau (1997)] on industrial blends (PP-PA6 blends with copolymers as compatibilizers) gave satisfying correlations. The influence of compatibilizers is to be studied next.

\section{A. Materials and preparation}

The blends contain polyisobutylene (PIB) and polydimethylsiloxane (PDMS) with comparable viscosities. The PIB was purchased from BP Chimie and the PDMS from Rhône-Poulenc. Their main characteristics at a temperature of $20^{\circ} \mathrm{C}$ are presented in Table 1. $\mathrm{Mn}(\mathrm{Mw})$ are respectively the number (weight) average molecular weight. PIB and PDMS are immiscible. In addition, their refraction indexes are not so different to allow visualization of one component in the other and vice-versa, as was mentionned by Grizzuti 
and Bifulco (1997) who used a very similar system to study break-up and coalescence. The interfacial tension between PIB and PDMS is about $3 \mathrm{mN} / \mathrm{m}$ at ambient temperature.

\begin{tabular}{|l|c|c|}
\hline & $\begin{array}{c}\text { PDMS } \\
\text { (Rhodorsil } \\
\mathbf{4 7 V 6 0 0 0 0 )}\end{array}$ & $\begin{array}{c}\text { PIB } \\
\text { (Parapol } \\
\mathbf{9 5 0})\end{array}$ \\
\hline $\mathrm{M}_{\mathrm{n}}(\mathrm{g} / \mathrm{mol})$ & 62400 & 932 \\
\hline $\mathrm{M}_{\mathrm{W}}(\mathrm{g} / \mathrm{mol})$ & 116500 & 1472 \\
\hline Polydispersity index & 1.87 & 1.58 \\
\hline$\eta_{0}($ Pa.s) & 65 & 58 \\
\hline Density $\rho\left(\mathrm{g} / \mathrm{cm}^{3}\right)$ & 0.978 & 0.881 \\
\hline Interfacial tension $\sigma(\mathrm{mN} / \mathrm{m})$ & \multicolumn{2}{|c|}{3.0} \\
\hline
\end{tabular}

Table 1. General properties of the two polymers at $20^{\circ} \mathrm{C}$. Viscosities from Carrimed CSL rheometer, PDMS data from Rhône-Poulenc, PIB density and molecular distribution (GPC) from BP-Chimie, $\sigma$ measured by the authors.

The thermal characteristics of both polymers at $20^{\circ} \mathrm{C}$ are given in Table 2 . Thermal properties are taken into account in the expressions of the complex coefficents $\mathrm{A}_{0}$ and $\mathrm{A}_{1}$ [Verdier and Piau (1997)] which appear in Eq. (2) giving the velocity of propagation and attenuation of longitudinal waves.

\begin{tabular}{|l|c|c|}
\hline & PDMS & PIB \\
\hline Specific heat at constant pressure: $\mathrm{C}_{\mathrm{p}}(\mathrm{J} / \mathrm{kg} / \mathrm{K})$ & 1600 & 2140 \\
\hline Thermal conductivity: $\tau(\mathrm{J} / \mathrm{m} / \mathrm{s} / \mathrm{K})$ & 0.16 & 0.13 \\
\hline Specific heat ratio: $\gamma(\mathrm{Cp} / \mathrm{Cv})$ & 1.05 & 1.06 \\
\hline Thermal dilatation: $\beta\left(\mathrm{K}^{-1}\right)$ & $5.410^{-4}$ & $6.810^{-4}$ \\
\hline Glassy temperature $\operatorname{Tg}\left({ }^{\circ} \mathrm{C}\right)$ & -123 & -68 \\
\hline
\end{tabular}

Table 2. Thermal properties of the two polymers at $20^{\circ} \mathrm{C}$ (Van Krevelen 1990).

To prepare a blend, the two polymers are first weighed and mixed slowly for about $2 \mathrm{mn}$ until a creamy mixture is obtained. After degassing, it was checked under the microscope that the blends produced by this technique are free of air bubbles, which would make drastic changes in the acoustical parameters. All blends were prepared according to the same procedure and the experiments were reproduced several times. These systems without compatibilizers are known to be unstable, and coalescence phenomena are important [Grizzuti and Bifulco (1997)], especially at high concentrations. So ultrasonic measurements and optical microscopy had to be carried out simultaneously within a short delay (a few minutes) after preparation of the blend.

\section{B. Optical microscopy}


The morphology of the blends which are melts at room temperature was examined under an optical microscope (Leica DM LM) between a glass plate and a micro cover glass. Some of the typical blend morphologies are presented in Fig. 1 for different concentrations $\phi$, where

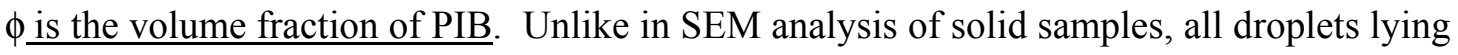
in the field depth of the microscope (around $40 \mu \mathrm{m}$ here) appear on the photographs, with clear droplets in front and others in the background. The size distribution and the average radius $\mathrm{R}_{\mathrm{a}}$ were determined by image analysis (NIH software) from the contrast between the different areas on the photographs. Figure 2 gives the results obtained for the average radius $\mathrm{R}_{\mathrm{a}}$ of PIB domains $(\phi<0.5)$ and PDMS domains $(\phi>0.5)$ respectively.

a)

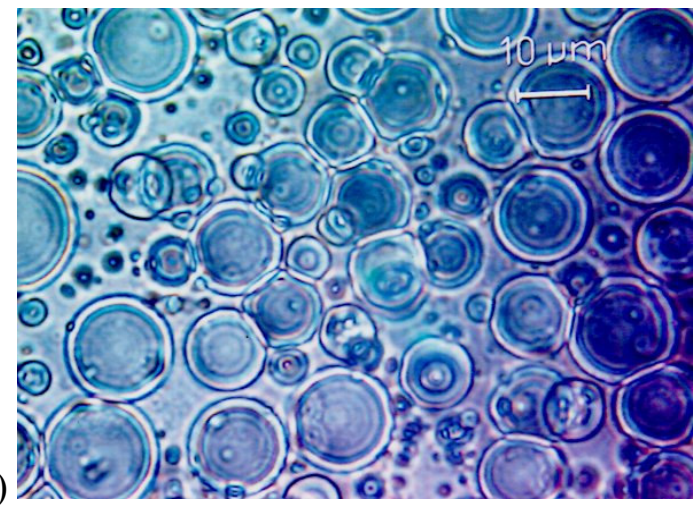

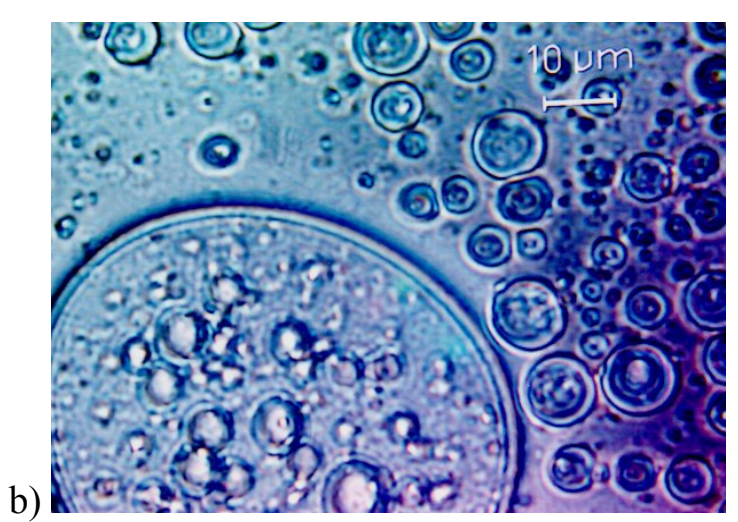

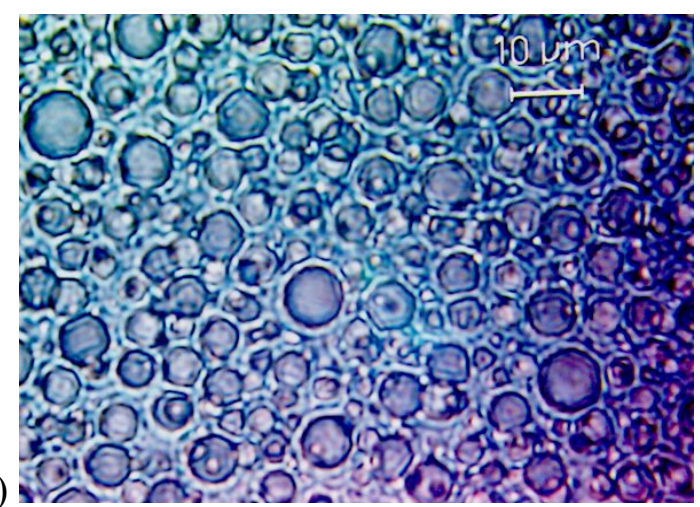

b)

c)

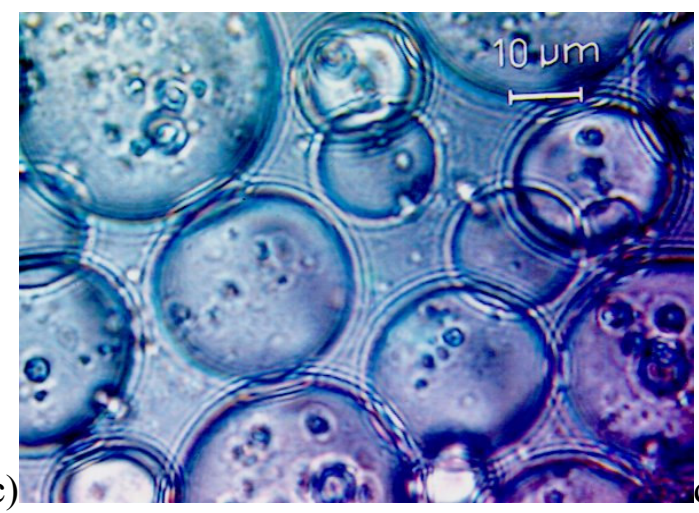

Fig.1. Optical microscopy a) $\phi=0.3 \quad \mathrm{R}_{\mathrm{PIB}}=6.5 \mu \mathrm{m} \quad$ b) $\phi=0.5 \quad$ Composites droplets
c) $\phi=0.6 \quad R_{P D M S}=13.5 \mu \mathrm{m}$
d) $\phi=0.8 \quad R_{P D M S}=3.5 \mu \mathrm{m}$

Optical microscopy shows that the minor phase when diluted (respectively PIB for $\phi=0.1$ and 0.2, and PDMS for $\phi=0.8$ and 0.9 ) was clearly dispersed in the matrix in the form of small droplets. The average droplet radius ranges between 3 and $5 \mu \mathrm{m}$, as seen for example in Fig. 1d $(\phi=0.8)$. For the cases $\phi=0.3$ [Fig. 1(a)] and 0.7, increased coalescence occurs 
during mixing and larger domains (PIB or PDMS) appear. Between $\phi=0.4$ and $\phi=0.6$ [Fig. 1(c)], a transition zone appears. As explained by Favis et al. (1987) in the case of PP/PC uncompatibilized blends, co-continuous phases or composite-droplet morphology of the dispersed phase may be observed in this concentration range. Figure $1(b)(\phi=0.5)$ shows an example of composite droplets where droplets of PDMS are enclosed inside larger droplets of PIB which are themselves included in the PDMS matrix. .

\section{Ultrasonic system: Longitudinal waves (transmission technique)}

Due to the high level of attenuation in PIB, a transmission technique is used for all samples, the blend to be analyzed being placed in a measurement cell between two identical ultrasonic transducers, one which acts as an emitter and the other as a receiver. The ultrasonic velocity $v_{L}$ and the attenuation coeffficient $\alpha_{L}(\mathrm{~dB} / \mathrm{m})$ through the sample are given by :

$$
\mathrm{v}_{\mathrm{L}}=\mathrm{d} / \Delta \mathrm{t}, \quad \alpha_{\mathrm{L}}=(20 / \mathrm{d}) \log (\mathrm{A} / \mathrm{B}),
$$

where $d$ is the length of the slit containing the sample, $\Delta t$ the time for the ultrasonic pulse (made of several sinusoids) to travel this distance, A the amplitude of the incident pulse (inside the sample) and B the amplitude of the pulse which has traveled through the material and impinges on the receiving transducer. PDMS is used as a calibrating material, with well-known ultrasonic characteristics [Verdier et al. (1998)]. A and B are deduced from the measurements by taking into account the contribution of the transmission coefficients (related to the impedance ratios) at the different interfaces.

Well-defined echoes are obtained as the pulse frequency used is close to the resonant frequency of the transducer $(2.4 \mathrm{MHz})$. Experiments are carried out on eleven fluids ranging from $\phi=0$ to $\phi=1$, by steps of 0.1 in concentration.

\section{Ultrasonic system : Shear waves (impedance technique)}

The method employed to measure both $\mathrm{G}^{\prime}$ and $\mathrm{G}^{\prime \prime}$ is based upon the measurement of the complex reflection coefficient of a shear wave at a solid-polymer interface. This method, developed by Mason et al. (1949), has been used previously by the authors [Longin et al. (1998)]. The principle is the following : a shear transducer is used as an emitter-receiver,

fixed on the oblique side wall of a piece of quartz (real impedance $\mathrm{Z}_{\mathrm{q}}$ ). The polymer sample 
is located on top of the quartz and the wave pulse impinges on this horizontal plane interface at an angle $\Phi$. The pulse is reflected, continues its way in the quartz until it hits the opposite side wall. Then it comes back to the horizontal interface and finally reaches the transducer. The shear impedance of the sample (or blend) is determined by comparing the nth echoes returning to the transducer, in the case without polymer (amplitude $A_{n}$ ) and with polymer (amplitude $\mathrm{B}_{\mathrm{n}}$ ). Large enough $\mathrm{n}$ are chosen to obtain a good precision. Let $\Delta \mathrm{t}$ be the time difference between the nth echoes and by $B_{n} / A_{n}$ their amplitude ratio. The following parameters $r$ and $\theta$ are computed first:

$$
r=\left(\frac{B_{n}}{A_{n}}\right)^{1 / 2 n} \quad \text { and } \quad \theta=\frac{\omega \Delta t}{2 n}
$$

The complex impedance $\mathrm{Z}_{\mathrm{S}}$ * and the complex shear moduli $\mathrm{G}_{\mathrm{b}}$ * of the polymer blend are derived from Eq. (14) :

$$
Z_{\mathrm{S}}^{*}(\omega)=Z_{\mathrm{q}} \cos \Phi \frac{1-\mathrm{r}^{2}+2 \mathrm{ir} \sin \theta}{1+2 \mathrm{r} \cos \theta+\mathrm{r}^{2}}=\left(\rho \mathrm{G}_{\mathrm{b}}{ }^{*}(\omega)\right)^{1 / 2}
$$

\section{RESULTS AND DISCUSSION}

\section{A. Longitudinal waves}

The velocity of propagation $v_{L}$ and attenuation $\alpha_{L}$ were determined at $20^{\circ} \mathrm{C}$ for longitudinal waves, at a frequency $\mathrm{f}=2.4 \mathrm{MHz}$, using the transmission technique described above . The measurements could be carried out at a different temperature, provided that a precise temperature control system both on the microscope plate and in the ultrasonic cell is used. Technical limitations in temperature and frequency are related to the transducer characteristics and to the level of attenuation obtained in the blend at lower temperatures or higher frequencies. The method used for the calculations is outlined below. 


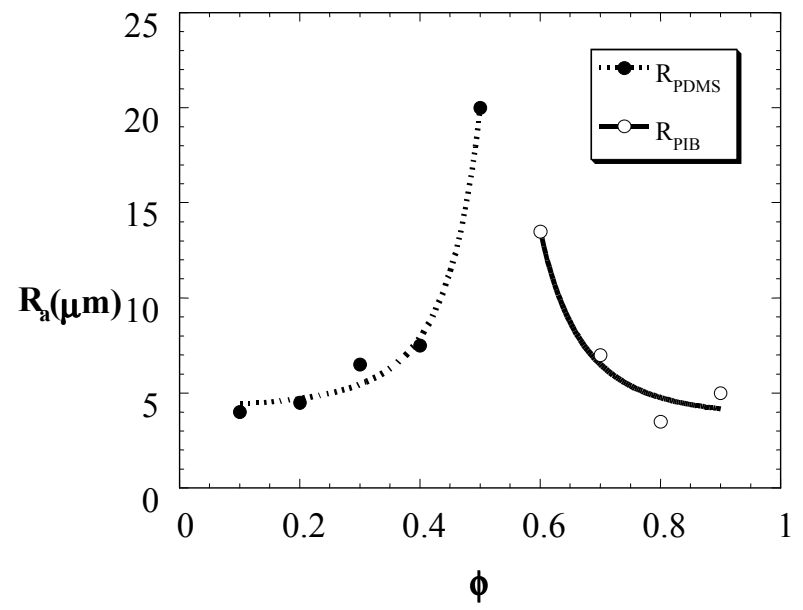

Fig. 2. Average droplet radius $R_{a}$, as a function of volume fraction $\phi$ of PIB in PDMS/PIB incompatible blends.

For each blend, microscopic observations and ultrasonic measurements were carried out at the same time. The different radii were determined from the optical microscopy clichés and averaged from photographs showing a larger number of domains to give the mean radius $R_{a}$ (Fig. 2) to be used for the theoretical calculations. The values of $v_{L}$ and $\alpha_{L}$ are shown in Figs. 3-4.

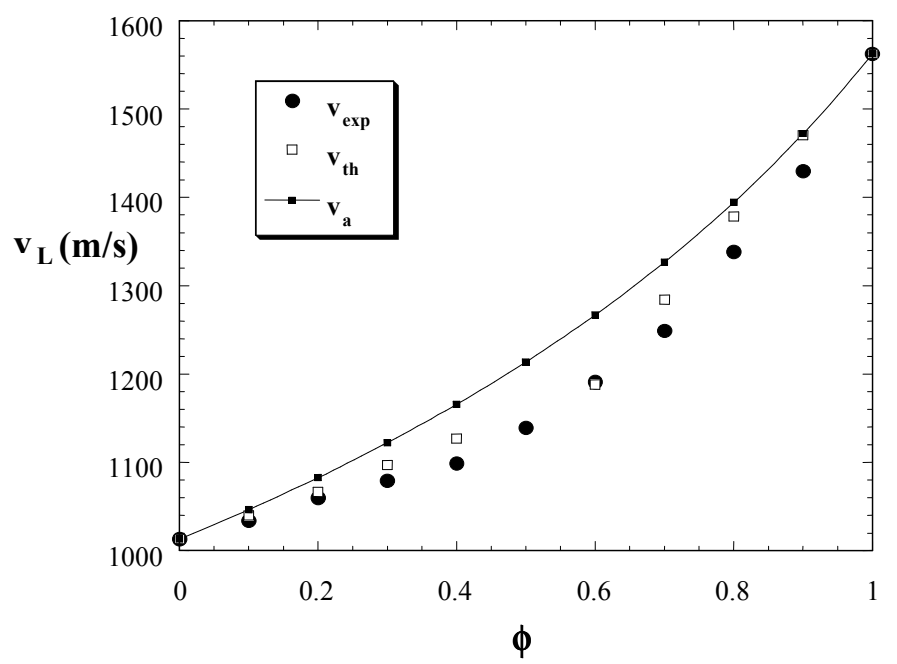

Fig. 3. Evolution of the velocity $v_{L}$ vs. blend composition $\left(\mathrm{f}=2.4 \mathrm{MHz}, \mathrm{T}=20^{\circ} \mathrm{C}\right)$ 


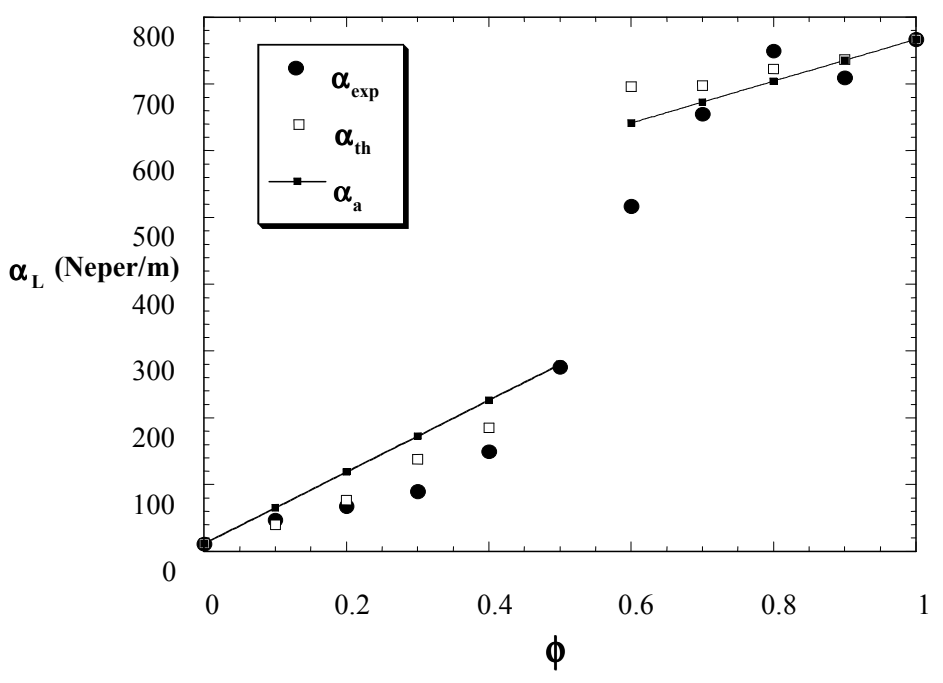

Fig. 4. Evolution of the attenuation $\alpha_{\mathrm{L}}$ vs. blend composition $\left(\mathrm{f}=2.4 \mathrm{MHz}, \mathrm{T}=20^{\circ} \mathrm{C}\right)$

For volume fractions between 0 and 40\%, PDMS is the matrix. Inversely, between 60 and $100 \%$ volume fraction, PDMS is the dispersed phase. At this frequency, the wavelength (in the range 400 to $600 \mu \mathrm{m}$ ) is much larger than the maximum droplet radius observed (20 $\mu \mathrm{m})$. Therefore the model corresponding to formula (2) applies for moderate concentrations, using the multiple scattering approach. This is also shown in Figs. 3-4. The average velocity $v_{a}$ of the blends defined by (3) (extended to the viscoelastic case by approximating $\kappa_{\mathrm{m}}{ }^{-1}$ and $\kappa_{\mathrm{d}}^{-1}$ by $\rho \mathrm{v}^{2}$ and $\rho \mathrm{v}_{\mathrm{d}}^{2}$ respectively) and the attenuation $\alpha_{\mathrm{a}}$ given by (4) are shown as a comparison. Between $40 \%$ and $60 \%$, the model is no longer relevant. For the sake of completeness, the model has been tested until $40 \%(\varepsilon=\phi)$. Similarly, when PIB is the matrix, the calculations range from $100 \%$ to $60 \%(\varepsilon=1-\phi)$.

For blends containing up to $30-40 \%$ of dispersed polymers, theoretical values of velocity and attenuation agree reasonably well with measured values. To explain the small deviations observed between the model and data, values of the average distance $\mathrm{L}$ between droplets and of the viscoelastic skin depth $\delta_{\mathrm{S}}$ are reported in Table 3, for the range of concentrations of interest. Indeed, at the frequency used, the thermal skin depth is of the order of 0.1 microns, so that the viscoelastic effects are dominant. Between 0 and $40 \%$, the distance between droplets decreases, and remains higher than half the viscoelastic skin depth in the PDMS matrix until $30 \%$ concentration, so that the model hypotheses may be 
considered roughly satisfied. On the contrary, between 60 and $90 \%$ of PIB, the droplet interspace is much smaller than the viscoelastic skin depth in the PIB matrix; this case corresponds to strong interactions between droplets, which are not really taken into account in the second order expansion of Eq. (2) in $\varepsilon^{2}$.

\begin{tabular}{|c|c|c|c|}
\hline $\begin{array}{c}\text { Volume } \\
\text { fraction } \\
\phi\end{array}$ & $\begin{array}{c}\text { Volume } \\
\text { fraction } \\
\varepsilon\end{array}$ & $\begin{array}{c}\text { Mean distance } \mathrm{L} \\
\text { between droplets } \\
(\mu \mathrm{m})\end{array}$ & $\begin{array}{c}\text { Radius } \mathrm{R}_{\mathrm{a}} \\
(\mu \mathrm{m})\end{array}$ \\
\hline 0.1 & 0.1 & 6.8 & 4 \\
\hline 0.2 & 0.2 & 4.2 & 4.5 \\
\hline 0.3 & 0.3 & 3.7 & 6.5 \\
\hline 0.4 & 0.4 & 2.5 & 7.5 \\
\hline 0.5 & - & - & 20 \\
\hline 0.6 & 0.4 & 4.5 & 13.5 \\
\hline 0.7 & 0.3 & 4.0 & 7 \\
\hline 0.8 & 0.2 & 3.3 & 3.5 \\
\hline 0.9 & 0.1 & 8.5 & 5 \\
\hline
\end{tabular}

Table 3. Mean distance between droplets $L=2 \mathrm{R}_{\mathrm{a}}\left\{(0.64 / \varepsilon)^{1 / 3}-1\right\}$, to be compared with viscoelastic skin depths : $\delta_{\mathrm{S}}=7.3 \mu \mathrm{m}$ (PDMS matrix), $\delta_{\mathrm{S}}=15.2 \mu \mathrm{m}$ (PIB matrix) from Eq. (6)

In the present work, it is not attempted to model the longitudinal wave propagation through composite droplets but this could be of interest. In fact, when phase inversion occurs (around 50\% volume concentration), a sudden change (Fig. 3) of the slope of $v_{L}$ is visible after an almost linear variation from 0 to $40 \%$. This phenomenon can also be observed by inspection of the attenuation (Fig. 4) which undergoes a rapid change, due to the high attenuation ratio between PDMS and PIB.

The high level of attenuation observed in PIB (roughly sixty times the value in PDMS) at 2.4 $\mathrm{MHz}$ and $20^{\circ} \mathrm{C}$ is related to the high value of the bulk viscous modulus $\mathrm{K}$ " (about $310^{8}$ $\mathrm{Pa}$, that is one thousand times the value for PDMS). This is related to the fact that the polymer glassy temperature is much lower in the case of PDMS (see Table 2). Values of the same order or higher have been reported for $\mathrm{K} "$ in the Megahertz range for other polymers such as PS, PMMA [Kono (1960)] or styrene-butadiene rubber [Wada et al. (1960)]. The 
shear moduli $\mathrm{G}^{\prime \prime}$ are also of the order $10^{8} \mathrm{~Pa}$ for these products, whereas values of the order $10^{6}-10^{7} \mathrm{~Pa}$ are obtained in PIB and PDMS (Fig. 5 a and b).
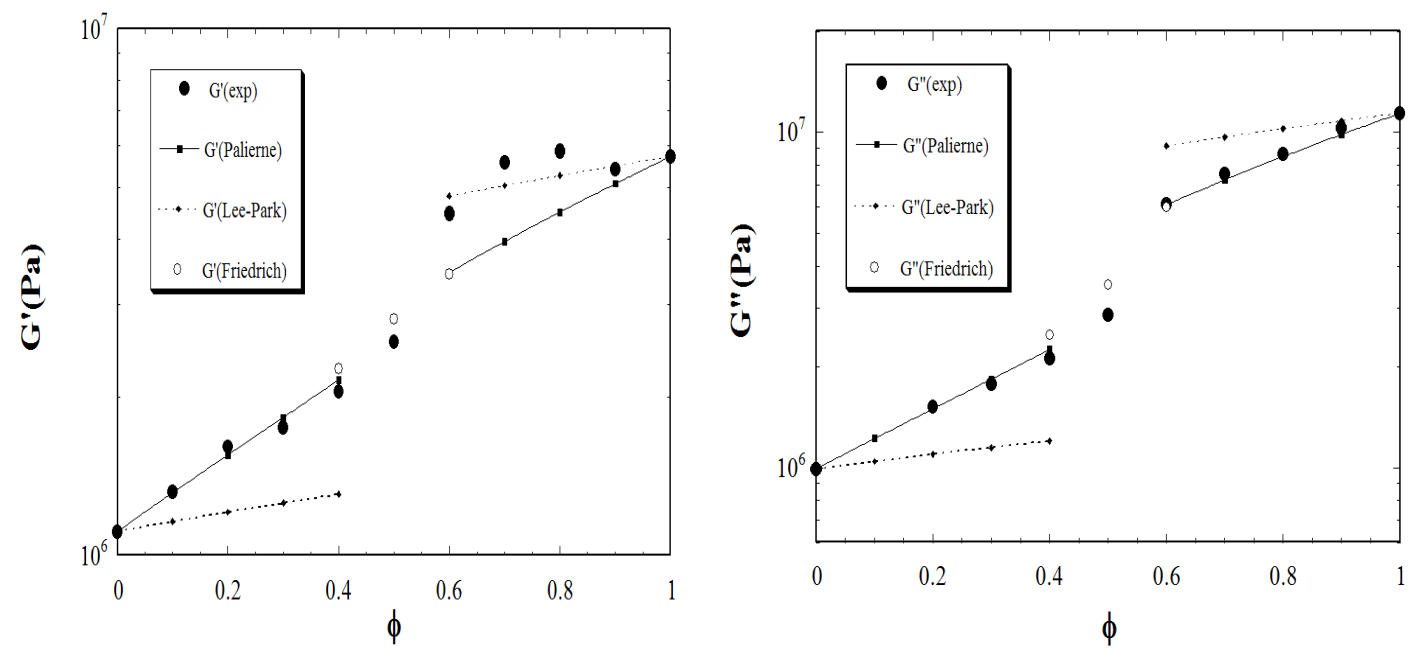

Fig. 5. Linear viscoelastic moduli a) $\mathrm{G}^{\prime}$; b) $\mathrm{G}^{\prime}$ ' as a function of volume fraction $\phi$ of PIB in PDMS/PIB blends. Comparison between experimental data and models predictions $\left(\mathrm{f}=2.4 \mathrm{MHz}, \mathrm{T}=20^{\circ} \mathrm{C}\right)$.

The ability of the present technique to monitor phase inversion as well as the influences of the radius and concentration in the blend arises as an interesting conclusion. The influence of the radius is inherent to the model as can be seen in formula (2) even if it does not clearly appear on the graphs. Similar results showing phase inversion in the case of solid systems have been obtained by Shaw and Singh (1987) for ethylene-propylene-diene blends and Sidkey et al. (1991) for rubber blends, but phase inversion has never been observed before in the case of melts via ultrasonic measurements .

\section{B. Shear waves}

All experiments were performed at $2.4 \mathrm{MHz}$ and a temperature of $20^{\circ} \mathrm{C}$, like in the case of longitudinal waves. The same eleven fluids have been studied. Results are reported in Fig. 5. The measurement starting time is the same as the one used for longitudinal measurements, and the morphology is the associated one.

The procedure used for the theoretical calculations is also the same: the polymer matrix is PDMS when $\phi$ ranges from 0 to 0.4 or PIB when $\phi$ ranges from 0.6 to 1 . 
The Palierne model seems to describe rather well the rheological behavior of the shear modulus $\mathrm{G}^{*}$ of the PIB/PDMS blends at high frequencies. The theoretical curves show a good agreement with the experimental data for the viscous modulus G". For the elastic modulus G', above a certain concentration in PIB, namely $60 \%$, the theoretical curves fall below the experimental points.

The Lee-Park model underestimates both viscoelastic shear moduli when the PDMS is the matrix. When the PIB composes the matrix, the blend theoretical loss modulus is too high. For the elastic modulus, correct agreement is obtained, better than in the case of the Palierne model, with no obvious explanation. PDMS and PIB were chosen with close zero shear viscosities, but their elastic and viscous shear moduli are quite different (an order of magnitude of variation). This discrepancy at high frequencies $(\phi>0.6)$ for the Lee-Park model has also been noticed by Lacroix et al. (1997) for the loss modulus of blends with low viscosity ratios. Figure 5 shows these predictions.

Despite the high contrast between the viscoelastic properties of the components, the Palierne model describes correctly the viscous moduli of most of the mixtures tested, even when composite droplets are present. The discrepancies observed could be partly explained by the fact that the morphology does influence the results at high frequencies.

The Palierne and Lee-Park models may provide good descriptions at moderate frequencies but still are not quite capable to give the best descriptions in the high frequency regions. A model of shear wave propagation in two-phase viscoelastic media, if adapted to such polymeric fluids, could give access to the high frequency range. Its extension to short wave-lengths would constitute a whole new problem.

Data obtained for volume fractions 40, 50, and $60 \%$ are also compared to the Friedrich model. We make use of Eqs. (10)-(11). From our photographs, $\phi_{1}$ can be determined rather well and we find that $\phi_{3}$ is quite small. Then mass conservation gives $\phi_{2}=\left(\varepsilon-\phi_{3}\right) /\left(1-\phi_{1}\right)$. This gives rise to the following data:

$$
\begin{aligned}
& * \phi=40 \% \text {, PDMS matrix, } \phi_{1}=15 \%, \phi_{2}=46.5 \%, \phi_{3}=0.5 \% \\
& * \phi=50 \% \text {, PDMS matrix, } \phi_{1}=25 \%, \phi_{2}=66 \%, \phi_{3}=0.5 \% \\
& * \phi=60 \% \text {, PIB matrix, } \phi_{1}=3 \%, \phi_{2}=40.7 \%, \phi_{3}=0.5 \%
\end{aligned}
$$


Using these numbers, we were able to determine $\mathrm{G}_{\mathrm{b}}{ }^{*}(\omega)$ for the three mentioned cases. This is shown in Figs. 5 (a) and 5 (b). The rapid upturn seen from our experimental data between $50 \%$ and $60 \%$ volume concentration validates the choice of PDMS as the matrix for the $50 \%$ case. The theoretical points lie between the two branches drawn from Palierne's theory and are close to the values obtained experimentally, which shows the relevance of the model. The comparison may be improved, but due to the difficulties inherent to the image analysis technique, we may conclude that satisfactory agreement is found using Friedrich's model, especially for the loss modulus G" (similarly to the Palierne's model).

\section{CONCLUSION}

The ability of the longitudinal wave propagation model adapted to viscoelastic emulsions is confirmed in the case of PDMS/PIB melts, even for rather high concentrations. For shear waves, elastic and loss moduli deduced from the measured complex impedance can also be relatively well described by the Palierne model and its extension to composite droplets.

This system is very interesting because optical microscopy has been performed together with longitudinal and shear wave measurements at all concentrations. Therefore it will be possible to test other rheological models based on the microstructural characteristics of the system.

This non intrusive technique allows to correlate the evolution of the size of the inclusions with the parameters measured at ultrasonic frequencies. It can be a very useful tool for controlling the mixing of polymers during the preparation of blends. 


\section{References}

Allegra, J. R., and S. A. Hawley, "Attenuation of sound in suspensions and emulsions: theory and experiments," J. Acoust. Soc. Am. 51, 1545-1564 (1972).

Arman, J., "Dispositif de mesure de la vitesse de propagation et du coefficient d'atténuation d'ultrasons dans les polymères", Acustica 43, 212-216 (1979).

Bousmina, M., "Effect of interfacial tension on linear viscoelastic behavior of immiscible polymer blends," Rheol. Acta. 38, 251-254 (1999).

Brahimi, B., A. Ait-Kadi, A. Ajji, R. Jérôme, and R. Fayt, "Rheological properties of copolymer modified polyethylene/polystyrene blends," J. Rheol. 35, 1069-1091 (1991).

Bridge, B., Cheng, K.H, "Non-destructive ultrasonic evaluation of $\mathrm{CaCO}_{3}$-filled polypropylene mouldings", J. Materials Sci. 22, 3118-3128 (1987).

Doi, M., and T. Ohta, "Dynamics and rheology of complex interfaces," J. Chem. Phys. 95, 1242-1248 (1991).

Epstein, P. S., and R. R. Carhart, "The absorption of sound in suspensions and emulsions. I. Water fog in air," J. Acoust. Soc. Am. 25, 553-565 (1953).

Fahrländer, M., and C. Friedrich, "Rheological properties of polymer blends with spherein-sphere morphology," Rheol. Acta 38, 206-213 (1999).

Favis, B. D., J .P. Chalifoux, and P. Van Gheluwe, S. P. E. Techn. Pap. 33, 1326 (1987).

Friedrich, C., W. Gleinser, E. Korat, D. Maier, and J. Weese, "Comparison of sphere-size distributions obtained from rheology and transmission electron microscopy in PMMA/PS blends," J. Rheol. 39, 1411-1425 (1995).

Graebling, D., and R. Müller, "Rheological behavior of polydimethylsiloxane /polyoxyethylene blends in the melt. Emulsion model of two viscoelastic liquids," J. Rheol. 34, 193-205 (1990).

Graebling, D., R. Müller, and J. F. Palierne, "Linear viscoelastic behavior of some incompatible polymer blends in the melt. Interpretation of data with a model of emulsion of viscoelastic liquids," Macromolecules 26, 320-329 (1993).

Gramespacher, H., and J. Meissner, "Interfacial tension between polymer melts measured by shear oscillations of their blends," J. Rheol. 36, 1127-1141 (1992).

Grizzuti, N., and O. Bifulco, "Effects of coalescence and breakup on the steady-state morphology of an immiscible polymer blend in shear flow," Rheol. Acta 36, 406-415 (1997).

Harker A.H. and J.A.G. Temple, "Velocity and attenuation of ultrasound in suspensions of particles in fluids," J. Phys. D: Appl. Phys. 21, 1576-1588 (1988)

Kaatze, U., C. Trachimov, R. Pottel, and M. Brai, "Broadband study of the scattering of ultrasound by polystyrene-latex-in-water suspensions," Ann. Physik 5, 13-33 (1996)

Kono R., "The dynamic bulk viscosity of polystyrene and polymethyl methacrylate," J. Phys. Soc. Japan 15, 718-725 (1960) 
Lacroix, C., M. Aressy, and P. J. Carreau, "Linear viscoelastic behavior of molten polymer blends: a comparative study of the Palierne and Lee and Park models," Rheol. Acta 36, 416-428 (1997).

Lacroix, C., M. Grmela, and P. J. Carreau, "Relationships between rheology and morphology for immiscible molten blends of polypropylene and ethylene copolymers under shear flow," J. Rheol. 42, 41-62 (1998).

Lee, H. M., and O. O. Park, "Rheology and dynamics of immiscible polymer blends," J. Rheol. 38, 1405-1425 (1994).

Lloyd, P., and M. V. Berry, "Wave propagation through an assembly of spheres. Relations between different multiple scattering theories," Proc. Phys. Soc. 91, 678-688 (1967).

Longin, P. Y., C. Verdier, and M. Piau, "Dynamic shear rheology of high molecular weight Polydimethylsiloxanes: comparison of rheometry and ultrasound," J. Non Newtonian Fluid Mech. 76, 213-232 (1998).

Mason, W. P., W. O. Baker and H. J. McSkimin, "Measurements of shear elasticity and viscosity of liquids at ultrasonic frequencies," Phys. Rev. 75, 936-947 (1949).

McClements, D. J. and M. J. W. Povey, "Ultrasonic velocity as a probe of emulsions and suspensions," Adv. Colloid Int. Sci. 27, 285-316 (1987).

McClements, D. J. and M. J. W. Povey, "Scattering of ultrasound by emulsions," J. Phys. D: Appl. Phys. 22, 38-47 (1989).

Oldroyd, J. G., "The elastic and viscous properties of emulsions and suspensions," Proc. R. Soc. London A 218, 122-132 (1953).

Palierne, J. F., "Linear rheology of viscoelastic emulsions with interfacial tension," Rheol. Acta 29, 204-214 (1990).

Piau, M. and C. Verdier, "On-line ultrasonic characterization of polymer blends," Proc. XIIth Int. Congr. on Rheology, 43 (1996).

Scholz, P., D. Froelich, and R. Müller, "Viscoelastic properties and morphology of twophase polypropylene/polyamide 6 blends in the melt. Interpretation of results with an emulsion model," J. Rheol. 33, 481-499 (1989).

Shaw, S. and R. P. Singh, "Study of compatibility of polystyrene with ethylene-propylenediene rubber," Eur. Polym. J. 23, 547-550 (1987).

Sidkey, M.A., A. M. Abd El Fattah, A. A. Yehia and N. S. Abd El All, "Ultrasonic investigation of some rubber blends," J. Appl. Polym. Sci. 43, 1441-1449 (1991).

Urick, R. J., "The absorption of sound in suspensions of irregular particles," J. Acoust. Soc. Am. 20, 283-288 (1948).

Utracki, L. A., "Polymers alloys and blends: thermodynamics and rheology," New York: Hanser (1989).

Van Krevelen, D. W., "Properties of polymers," Elsevier, New-York, 3rd Ed. (1990).

Verdier, C., and M. Piau, "Analysis of the morphology of polymer blends using ultrasound," J. Phys. D: Appl. Phys. 29, 1454-1461 (1996). 
Verdier, C., and M. Piau, "Acoustic wave propagation in two-phase viscoelastic fluids: the case of polymer emulsions," J. Acoust. Soc. Am. 101, 1868-1876 (1997).

Verdier, C., P.Y. Longin and M. Piau, "Dynamic shear and compressional behaviour of polydimethylsiloxanes : ultrasonic and low frequency characterization' Rheol. Acta 37, 234-244 (1998)

Vinckier, I., P. Moldenaers, and J. Mewis, "Relationship between rheology and morphology of model blends in steady shear flow," J. Rheol. 40, 613-631 (1996).

Wada Y., H. Hirose, H. Umebayashi and M. Otomo, "Volume viscoelasticity of polymers and other highly dissipative materials," J. Phys. Soc. Japan 15, 2324-2334 (1960) 